\title{
Behaviour of turbulent transport in the vicinity of a magnetic island
}

\author{
E. Poli ${ }^{1}$, A. Bottino ${ }^{1}$, A. G. Peeters ${ }^{2}$ \\ ${ }^{1}$ Max-Planck-Institut für Plasmaphysik, EURATOM Association, D-85748 Garching, Germany \\ ${ }^{2}$ Centre for Fusion, Space and Astrophysics, Department of Physics, University of Warwick, \\ CV4 7AL Coventry, United Kingdom \\ e-mail: Emanuele.Poli@ipp.mpg.de
}

\begin{abstract}
The influence of a magnetic island on the behaviour of electrostatic turbulence in a tokamak is investigated numerically employing global gyrokinetic particle-in-cell simulations. Close to and inside the reconnected region, the shape of the turbulent eddies is changed; in particular, the transport at the island separatrix around the $O$-point is reduced. Low mode numbers in the energy spectrum of the potential disturbances, corresponding to the island perturbation, are amplified by nonlinear coupling with the microinstabilities. The temperature profile in the island region is determined numerically and its consistency with analytic estimates is discussed.
\end{abstract}

\section{Introduction}

The tearing mode $[1,2]$ is a resistive magnetohydrodynamic instability which leads to reconnection of the magnetic field within a layer around a given "resonant" surface. In a tokamak, tearing modes lead to the appearence of magnetic islands on low-order rational magnetic surfaces. Since within the island the magnetic field connects regions on both sides of the rational surface, the fast parallel streaming of the particles along the field lines leads to the consequence that a pressure gradient cannot be sustained inside the island separatrix. In today's tokamak experiments, the tearing mode is often driven unstable by the bootstrap current perturbation caused by the flattening of the pressure profile inside the island [3-5]. In this case the mode is called Neoclassical Tearing Mode (NTM). For a complete description of the dynamics of a NTM, it is therefore necessary to understand the interplay between the mode and the transport processes that determine the density and temperature profiles of the plasma. In a fusion reactor, the transport is to a large extent determined by microinstabilites leading to the formation of turbulent structures which enhance the particle and heat fluxes well above the neoclassical level. A magnetic island has a direct influence on the radial transport, since it provides a radial magneticfield component, thus leading to the appearence of a radial parallel transport, which is otherwise absent in tokamaks. Moreover, the above-mentioned flattening of the pressure profile inside the island drastically reduces the drive of the turbulence. Finally, the development and the shape of the turbulent structures can be modified by the helical field of the mode. The turbulence itself can affect the dynamics of magnetic islands, first of all providing "seed islands" in the presence of strong electromagnetic turbulence, and contributing to determining the pressure profile (and hence the level of bootstrap current) around the island separatrix, where perpendicular and parallel transport are supposed to be comparable (cf. Sec. 4).

From the previous considerations, it emerges that the topic we are discussing involves a wide range of time and scale lenghts, namely those characterizing the motion of the particles, those typical of the turbulent processes up to the evolution space- and timescales of the island and of the equilibrium itself. This makes a complete solution of the problem not affordable. Here, we will focus on the features of electrostatic turbulence in the presence of a prescribed island. Therefore, no evolution of the mode is retained in our numerical scheme, i. e. the island width has a fixed value and no island rotation is considered.

The problem of turbulent transport in the presence of an island is studied in this paper employing the global gyrokinetic particle-in-cell (PIC) code ORB5 [8]. The gyrokinetic equation is solved following the trajectories of an ensemble of markers moving according to the gyrokinetic equations of motion. The self-consistent electric field is calculated by solving the Poisson 
equation on a fixed spatial grid. In the simulations presented here, only electrostatic instabilities are considered. In this scheme, the inclusion of an island structure can be performed in a straightforward way by adding a small radial magnetic-field component in the equations of motion, which allows to account for the most important modification of particle orbits [9] (the details are explained in Sec. 2). It is noted that a gyrokinetic approach is needed not only to properly treat the dynamics of the microinstabilities, but also because finite-orbit effects can become essential for small islands and in any case around the separatrix $[10,11]$.

\section{The numerical scheme}

ORB5 provides a numerical solution to the gyrokinetic equations in the formulation of T. S. Hahm [12]. The distribution function is split into an analytically-known time-independent part $f_{0}$ and a perturbation $\delta f$ which is represented numerically by an ensemble of markers. These markers evolve in time according to the gyrokinetic equations of motion,

$$
\begin{aligned}
\frac{\mathrm{d} \mathbf{R}}{\mathrm{d} t}= & v_{\|} \mathbf{b}+\frac{1}{B_{\|}^{*}}\left[\frac{\mu B+v_{\|}^{2}}{\Omega_{c i}} \mathbf{b} \times \nabla B-\frac{v_{\|}^{2}}{\Omega_{c i}} \mathbf{b} \times(\mathbf{b} \times \nabla \times \mathbf{B})-\nabla\langle\phi\rangle_{g} \times \mathbf{b}\right], \\
\frac{\mathrm{d} v_{\|}}{\mathrm{d} t}= & -\mu\left[\mathbf{b}-\frac{v_{\|}}{B_{\|}^{*} \Omega_{c i}} \mathbf{b} \times(\mathbf{b} \times \nabla \times \mathbf{B})\right] \cdot \nabla B \\
& -\frac{q_{i}}{m_{i}}\left\{\mathbf{b}+\frac{v_{\|}}{B_{\|}^{*} \Omega_{c i}}[\mathbf{b} \times \nabla B-\mathbf{b} \times(\mathbf{b} \times \nabla \times \mathbf{B})]\right\} \cdot \nabla\langle\phi\rangle_{g}, \\
\frac{\mathrm{d} \mu}{\mathrm{d} t}= & 0,
\end{aligned}
$$

where $\mathbf{R}$ is the position of the gyrocentre, $v_{\|}$the velocity component along the magnetic field, $\mathbf{b}$ the unit vector along the magnetic field $\mathbf{B}, \mu$ the magnetic moment, $\Omega_{c i}$ the cyclotron frequency, $\langle\phi\rangle_{g}$ the perturbed potential (solution of the Poisson equation) averaged over the gyroperiod, $q_{i}$ and $m_{i}$ the particle's charge and mass, respectively, and $B_{\|}^{*}=B+\left(m_{i} / q_{i}\right) v_{\|} \mathbf{b} \cdot \nabla \times \mathbf{b}$. Since along the orbits $\mathrm{d} f / \mathrm{d} t=0, \delta f$ must obey the equation

$$
\frac{\mathrm{d}(\delta f)}{\mathrm{d} t}=-\frac{\mathrm{d} f_{0}}{\mathrm{~d} t}=-\mathbf{v} \cdot \nabla f_{0} .
$$

The perturbed potential is obtained as the solution of the Poisson equation

$$
\nabla^{2} \phi=4 \pi q_{i}\left\{n_{e}-\int\left[f+\frac{q_{i}}{m_{i} B}(\phi-\langle\phi\rangle) \frac{\partial f}{\partial \mu}\right] \delta(\mathbf{R}+\rho-\mathbf{r}) \mathrm{d}^{6} \mathbf{Z}\right\}
$$

where $\rho$ is a vector directed from the gyrocentre to the position of the particle and $\langle\phi\rangle$ is the flux-surface-averaged potential. The charge connected to each marker is assigned pointwise to a spatial mesh (charge assignment) in order to provide the source term for the Poisson equation. The computation of the gyroaveraged density follows an adaptive procedure, in order to have the same number of sampling points per arclength along the gyro-ring. Once the perturbed gyroaveraged charge density associated with each marker has been projected onto the splines, the equation for the components of the potential on this basis reduces to an algebraic matrix equation.

It should be added that the same spline basis is used to interpolate the radial magnetic-field perturbation, which is initially assigned on a grid. 

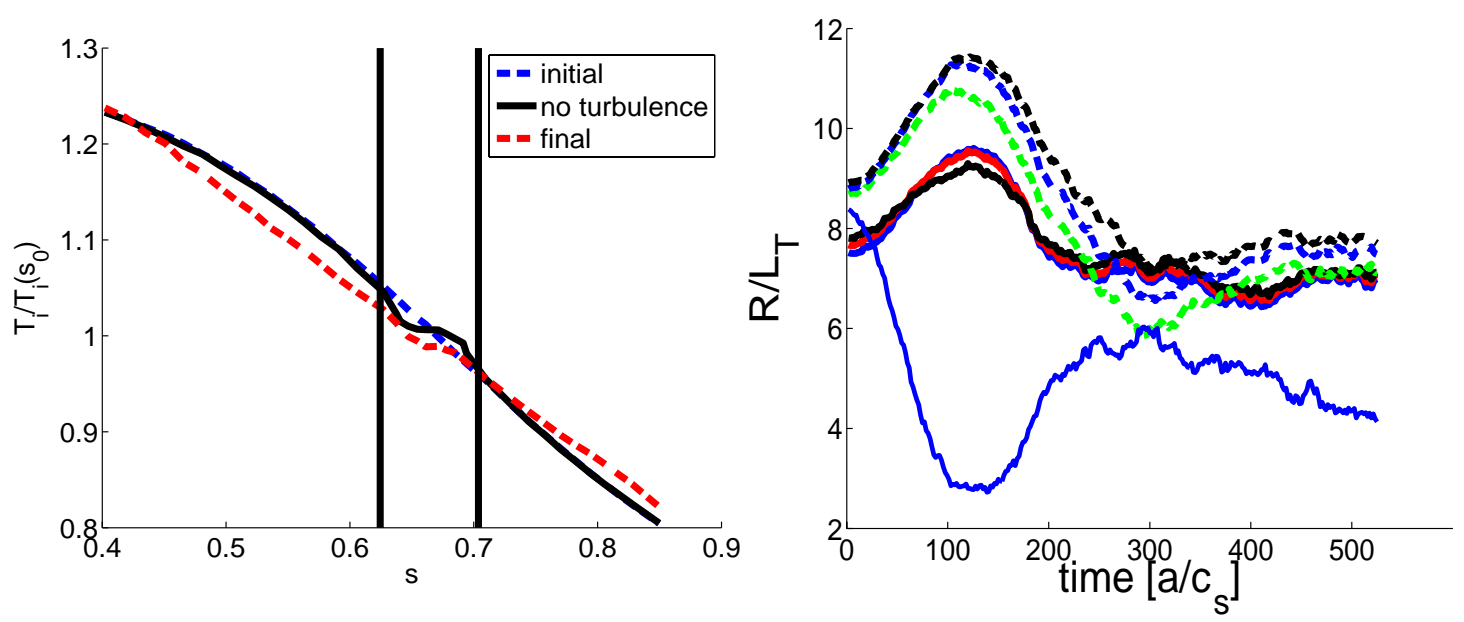

FIG. 1: Left: Typical temperature profile of a numerical simulation at different time slices. In this simulation, the ratio between the thermal ion banana width $w_{b}$ and the island width $W$ is about 0.4. Right: the evolution of the normalized inverse gradient length $R / L_{T}$ : solid lines correspond to radial positions around the inner island separatrix, dashed line to the outer separatrix. The lowest curve refers to the centre of the island.

\section{Magnetic islands}

In the presence of a magnetic island, the magnetic field can be represented as

$$
\mathbf{B}=\nabla \psi_{t} \times \nabla \xi / m+\nabla \varphi \times \nabla \Psi_{\mathrm{he}},
$$

where $\psi_{t}$ is the toroidal flux, $\xi=m \theta-n \varphi$ is the helical angle ( $\theta$ and $\varphi$ being the poloidal and toroidal angles, respectively, and $m$ and $n$ the poloidal and toroidal number characterising the island) and

$$
\Psi_{\mathrm{he}}=\psi-\frac{\psi_{t}}{q_{s}}+\alpha \cos \xi
$$

is the helical flux ( $\psi$ is the poloidal flux and the subscript $s$ denotes that a quantity is calculated at the resonant $(m, n)$ surface). If $\alpha=0$, it is easy to show that Eqs. $(6,7)$ reduce to the usual representation of the magnetic field. The last term of Eq.(7) describes the field perturbation due to the island, which is therefore $\tilde{\mathbf{B}}=\alpha \nabla \varphi \times \nabla \cos \xi=m \alpha \sin \xi \nabla \theta \times \nabla \varphi$ (here, $\alpha$ is approximated to be a constant). The new field component is directed along $\nabla \psi$. The helical flux introduced in Eq. (7) can be used to label the perturbed magnetic surfaces, as $\mathbf{B} \cdot \nabla \Psi_{\mathrm{he}}=0$. The most important consequence for the particle trajectories resulting from the new magnetic-field topology is that the motion parallel to the field includes now a radial component. This has been included in ORB5 by substituting $\mathbf{b} \rightarrow \mathbf{b}+\tilde{\mathbf{b}}$ (where $\tilde{\mathbf{b}}=\tilde{\mathbf{B}} / B$ ) in the first term of both Eq. (1) and Eq. (2). An important quantity which determines how fast the particles can stream along the island in the $\xi$-direction is the parallel wavevector $k_{\|}$, which is proportional to the distance from the rational surface and to the magnetic shear:

$$
k_{\|}=\frac{m}{q R} \frac{r-r_{s}}{L_{q}}=\frac{\varepsilon_{s} s_{s} n}{r_{s}^{2}}\left(r-r_{s}\right)
$$

where $1 / L_{q}=(1 / q) \mathrm{d} q / \mathrm{d} r$ is the inverse length characterizing the safety-factor profile, $\varepsilon$ is the inverse aspect ratio and $s$ the magnetic shear. 

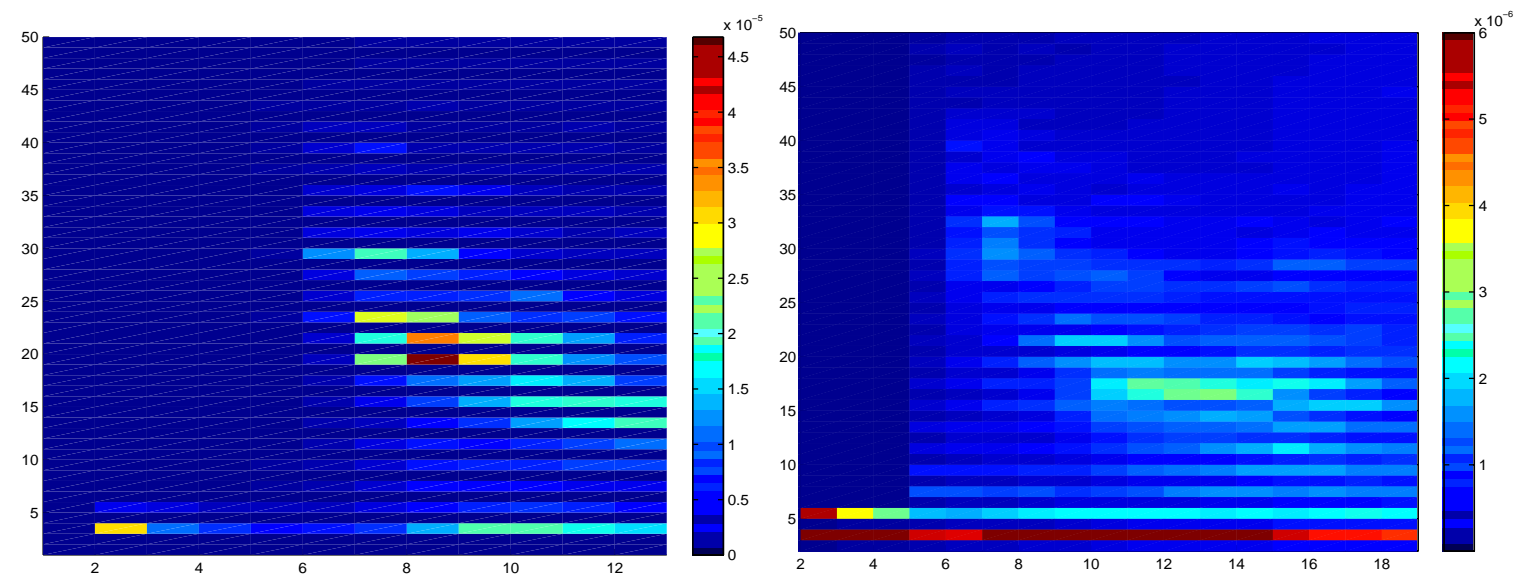

FIG. 2: Time evolution of the energy spectrum for moderate island width (left, only even modes retained) and large island width (right, longer run). On the $x$-axis is the time and on the y-axis is the toroidal mode number.

\section{Parallel and perpendicular transport close to the island separatrix}

As already pointed out in the Introduction, since the transport along the field lines is much larger than across the field, the pressure profile can be thought to be a function of the perturbed magnetic-flux label $\Psi_{\text {he }}$ introduced above. Under this assumption, the pressure gradient jumps from a finite value to zero when the island separatrix is crossed. However, the ratio between, say, the parallel and perpendicular heat conductivity in a tokamak is indeed very large (up to $\chi_{||} / \chi_{\perp} \approx 10^{9}-10^{10}$ ) but finite. As a consequence, a boundary layer appears around the island separatrix, along which the heat is transported from one side of the rational surface to the other $[6,7]$. The features of this process have been investigated solving the steady-state heat diffusion equation [6]

$$
\chi_{\|} \nabla_{\|}^{2} T+\chi_{\perp} \nabla_{\perp}^{2} T=0
$$

or, alternatively, ${ }^{1}$ the kinetic equation [7]

$$
v_{\|} \nabla_{\|} f=D_{\perp} \nabla^{2} f
$$

The critical width $w_{c}$ in which parallel and perendicular transport compete is obtained by equating the two terms of the previous equations. Thus in Eq. (9) we can estimate $\chi_{\|} k_{\|}^{2} \sim$ $\chi_{\perp} / w_{c}^{2}$, and assuming $r-r_{s} \sim w_{c}$ in Eq. (8), the scaling for $w_{c}$ turns out to be $w_{c} / r \sim$ $\left(\chi_{\perp} / \chi_{\|}\right)^{1 / 4}\left(1 / \varepsilon_{S} s_{S} n\right)^{1 / 2}$. The corresponding estimate derived from Eq. (10), namely $w_{c} \sim$ $\left(D_{\perp} / k_{\|} v_{\mathrm{th}}\right)^{1 / 2}$, can be reduced to the above if a parallel diffivity $D_{\|} \sim v_{\mathrm{th}} / k_{\|}$is introduced (see footnote) and again taking $r-r_{s} \sim w_{c}$ in Eq. (8). In the transition layer, the temperature is not a flux-surface function. The heat is found to be transported along the layer and to flow across the rational surface near the $X$-point [6]. The analysis of [7], moreover, predicts that the jump $\Delta f$ of the distribution function on both sides of the island should be proportional to the gradient $\mathrm{d} f / \mathrm{d} r$ at the island separatix, the proportionality factor being given by the width of the critical layer $w_{c} \simeq \sqrt{\chi_{\perp} / k_{\|} v_{\text {th }}}$.

In the next section, these picture is compared with the results of direct numerical simulations of turbulent transport. It has to be stressed that, in both approaches, the dependence of the

\footnotetext{
${ }^{1}$ It is noted that replacing conduction by convection, i. e. approximating in Eq.(9) the term $\chi_{\|} \nabla_{\|}^{2} T$ with $v_{\|} \nabla_{\|} T$ (which is in turn equivalent to estimating $\chi_{\|} \sim v_{\text {th }} / k_{\|}$[6]) one obtains an equation of the same form as Eq.(10).
} 
perpendicular (heat) diffusion coefficient on the radial coordinate $\psi$ and on the helical angle along the island $\xi$ has been neglected, in order to obtain an analytic solution of the starting equation.

\section{Numerical results}

The numerical simulations presented in this section have been performed for a tokamak with circular concentric flux surfaces, major radius $R_{0}=3.3 \mathrm{~m}$ and minor radius $a=0.47 \mathrm{~m}$. A flat density gradient is considered, the turbulent transport being caused by an electrostatic Ion-Temperature-Gradient (ITG) instability. As the proper computation of the flux-averaged ("zonal") potential with adiabatic electrons becomes extremely difficult in ORB5 if a magnetic island is present, the results presented here do not include zonal flows. No sources are present, so that the temperature profile relaxes according to the level of the heat flux. In order to avoid a too fast relaxation and ensure a "quasi-stationary" turbulent phase, a pretty small value of the normalized gyroradius $\rho_{\star} \equiv \rho / a=1 / 320$ has been taken. As this corresponds to a pretty low value for the ion temperature, the ion streaming along the island is not very fast. Typical values of $\chi_{\|} / \chi_{\perp}$ are therefore ${ }^{2}$ in the range $10^{6}-10^{7}$.

Since the initial temperature profile is a function of the constant unperturbed flux $\psi$, the turbulence is switched off in the inital phase of each run to allow the markers to move along the perturbed field lines, thus flattening the temperature profile in the island. During this phase, the temperature gradient increases outside the island because the flux surfaces are "compressed" with respect to the unperturbed equilibrium (without island). Subsequently, under the influence of turbulent transport the temperature gradient decreases, particularly fast at the end of the linear phase (overshoot). At the end of the run, a phase with almost constant temperature gradient in the island region is observed. An example of this evolution is shown in Fig. 1.
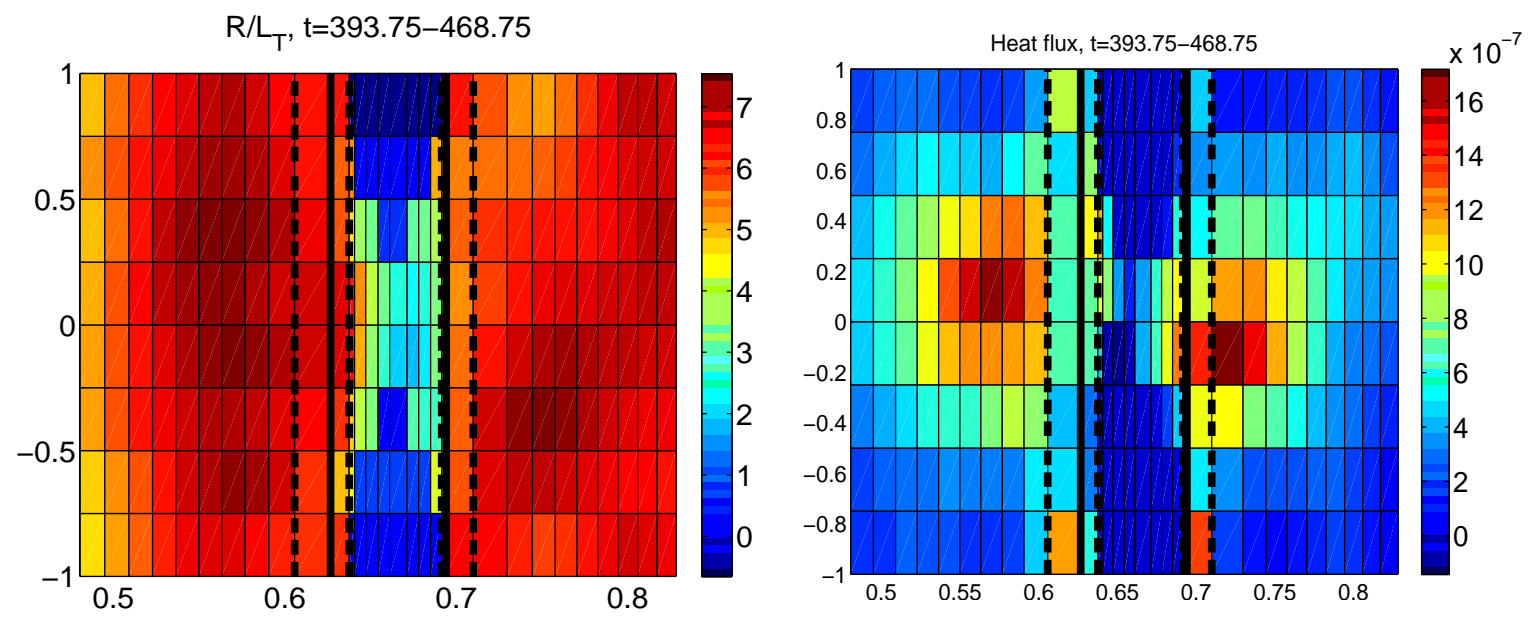

FIG. 3: Normalized inverse gradient length $R / L_{T}$ and the $\mathbf{E} \times \mathbf{B}$ heat flux, as a function of the radial coordinate $s=\sqrt{\psi}$ and of the helical angle $\xi / 2 \pi$, during the phase with fully-developed turbulence.

First of all, the numerical results concerning the development of the turbulence in the island region are discussed. An analysis of the energy spectrum of the turbulence shows that moving from the linear to the nonlinear phase the spectrum exhibits an inverse cascade to smaller mode numbers. In our simulations with a $(3,2)$ island, even toroidal numbers (multiples of the island mode number) are found to dominate with respect to the odd ones for large island widths

\footnotetext{
${ }^{2}$ Here, according to Ref. [6], we estimate $\chi_{\|} \sim v_{\text {th }} / k_{\|}$, see Sec. 4
} 
(cf. Fig. 2). Moreover, the low- $n$ island modes are amplified through nonlinear coupling with the turbulent modes. In our runs, seed $n=2$-harmonics arise during the turbulence-free phase of the run mentioned above; under experimental conditions, in general, the low- $n$ potential associated to the island rotation with respect to the plasma can interact nonlinearly with the fluctuating field of the microinstabilities. An analysis of the heat fluxes in the island region reveals that the $\mathbf{E} \times \mathbf{B}$ transport is stronger for values of $\xi$ corresponding to the island $O$-point, where the flux surfaces are closer to each other and the temperature gradient is therefore higher. However, this is true only up to a given distance from the island. Close the separatrix, the largest fluxes are found in the $X$-point region and the transport around the $O$-point is reduced. This result, which is found in both the linear and in the nonlinear phase, can be explained by the fact that the turbulent $\mathbf{E} \times \mathbf{B}$ eddies can develop across the resonant surface at the $X$-point position, whereas it seems that they are broken across the separatrix in the $O$-point region (cd. Fig. 4).
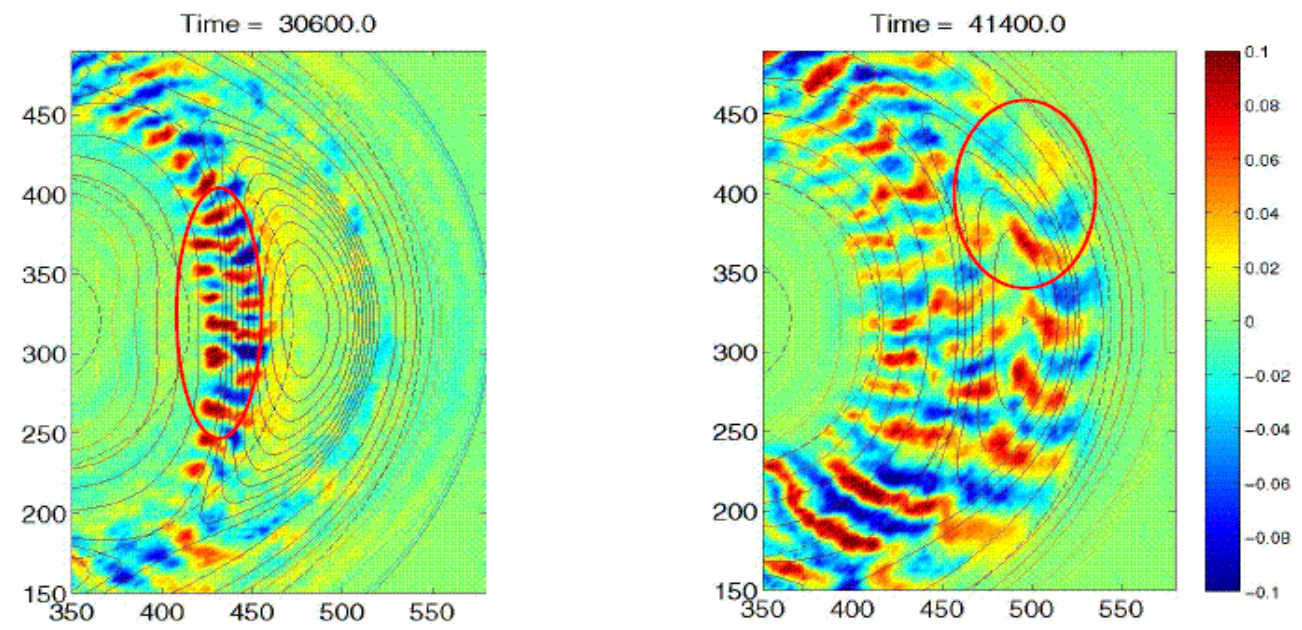

FIG. 4: Breaking of the eddies near the separatrix and their deformation inside the island.

In other words, cross-surface transport can apparently occur without undergoing the process of crossing the separatrix at the $O$-point and passing the rational surface at the $X$-point [6]. This can have an impact on the shape of the temperature profile across the island (see below). In the presence of zonal flows, the impact of elongated vortices across the $X$-point, however, could become less important.

In the simulations, the ratio between the radial $\mathbf{E} \times \mathbf{B}$ flux

$$
q_{E}=\int \frac{m v^{2}}{2} v_{E} \delta f \mathrm{~d}^{3} \mathbf{v}
$$

and the radial component of the parallel flux along the perturbed field lines

$$
q_{\|, r}=\int \frac{m v^{2}}{2} v_{\|} \tilde{b} \delta f \mathrm{~d}^{3} \mathbf{v}
$$

is calculated. As expected, inside the island separatrix there is a layer where these fluxes are of the same size. Depending on the strength of the turbulence, the ratio $q_{E} / q_{\|, r}$ can be above or below one. It is interesting to note that in the very centre of the island, this ratio can be one or two order of magnitude higher than at the separatrix. In the island centre, the parallel transport becomes less and less effective, since $k_{\|}$is proportional to the distance from the rational surface, whereas turbulent structures can in some case reach the $O$-point or be transported there by 

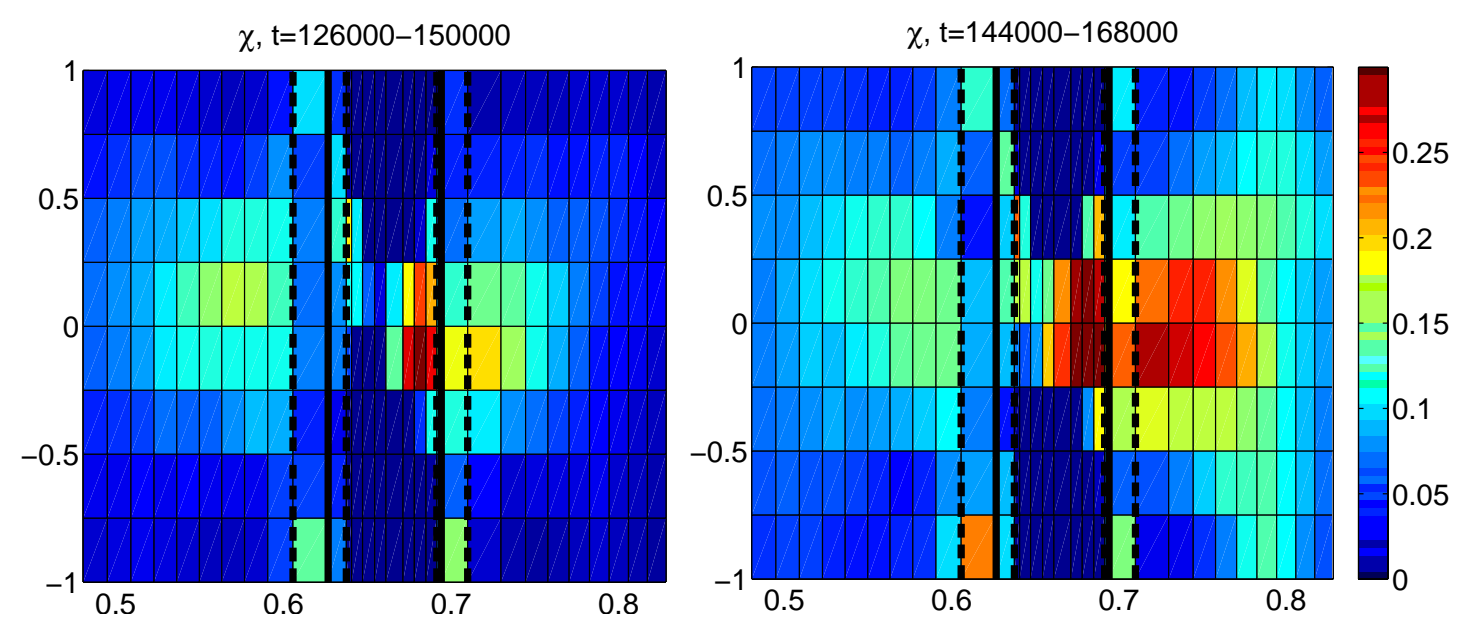

FIG. 5: Perpendicular thermal conductivity in gyro-Bohm units in the nonlinear phase for two different value of the initial logarithmic temperature gradient. The highest values are attained inside the island, since the temperature gradient is much smaller there. Coordinates as in Fig. 3.

the diamagnetic rotation of the instability. In any case, $q_{E} / q_{\|, r}$ can change significantly as a consequence of small variations of the background gradients.

The behaviour of the temperature profile in the numerical simulations is in qualitative agreement with the prediction obtained from an analytic solution [6] of the equation $\nabla \cdot \mathbf{q}=0$ summarized in Sec. 4. The width of this transition layer seems to be smaller than in the analytic estimate, probably because of the reduction of the $O$-point fluxes mentioned above. The suggested proportionality between the jump $\Delta T$ of the temperature profile on both sides of the island and the gradient $\mathrm{d} T / \mathrm{d} r$ at the island separatrix [7] is not confirmed by the simulations, where the product $w_{c} \mathrm{~d} T / \mathrm{d} r$ is found to increase faster than $\Delta T$ if the temperature gradient is increased.

One possible explanation for the results reported above, which contradict analytic estimates, is that the assumption of uniform heat conductivity is not verified in the simulations. Close to the island separatrix, $\chi_{\perp}$ displays a strong variation as a function of the radius and in particular of the helical angle, see Fig. 5. Moreover, $\chi_{\perp, X}$ and $\chi_{\perp, O}$ vary differently depending on plasma parameters, partcularly on the temperature gradient. Here, $\chi_{\perp}$ is calculated as the ratio between the $\mathbf{E} \times \mathbf{B}$ heat flux and the local ( $\xi$-dependent) temperature gradient.

\section{Conclusions}

PIC codes allow a conceptually straightforward implementation of a magnetic island through a modification of the equations of motion which take into account the presence of a (small) radial component of the magnetic field. In the simulations presented here, where adiabatic electrons were assumed and zonal flows were excluded, the temperature profile as determined by the turbulent transport is in qualitative agreement with the analytic predictions: the temperature is flat inside a sufficiently large island, is a flux function well outside it, with a transition layer located at the island separatrix. The detailed structure of this transition layer, however, is connected to the behaviour of the turbulence in the island region. In particular, the value of the thermal diffusivity is found to vary significantly in both the radial and the helical direction. It has been shown that when elongated eddies are present in the $X$-point region, a large part of the crosssurface transport takes place there. In many circumstances, the transport across the $O$-point region seems to be suppressed. The verification of the results presented here in the presence of zonal flows, employing kinetic electrons, is under development. 


\section{References}

[1] FURTH, H. P. et al., Physics of Fluids 6 (1963) 459.

[2] RUTHERFORD, P. H., Physics of Fluids 16 (1973) 1903.

[3] QU, W. X. et al., University of Wisconsin Plasma Report UWPR 85-5 (1985).

[4] CARRERA, R. et al., Physics of Fluids 29 (1986) 899.

[5] CHANG, Z. et al., Physical Review Letters 74 (1995) 4663.

[6] FITZPATRICK, R., Physics of Plasmas 2 (1995) 825.

[7] HAZELTINE, R. D. et al., Physics of Plasmas 4 (1997) 2920.

[8] JOLLIET, S. et al., Computer Physics Communications 177 (2007) 409.

[9] WHITE, R. B. et al., Physics of Fluids 26 (1983) 2958.

[10] WAELBROECK, F. L. et al., Physical Review Letters 87 (2001) 215003.

[11] JAMES, M. et al., Plasma Physics and Controlled Fusion 48 (2006) 1647.

[12] HAHM, T. S., Physics of Fluids 31 (1988) 2670. 